\title{
Bioequivalence Evaluation of Two Brands of Etoricoxib 120mg Tablets (Etoricoxib-SAJA \& ARCOXIA®) - in Healthy Human Volunteers
}

\author{
Omaima N. Najib ${ }^{1,2}$, Rana Hassan², Bassam Alwadi ${ }^{3}$, Nasir M. Idkaidek ${ }^{4}$ and Naji M. Najib² \\ ${ }^{1}$ College of Pharmacy, Philadelphia University, Amman, Jordan \\ ${ }^{2}$ International Pharmaceutical Research Center, Amman, Jordan \\ ${ }^{3}$ SAJA Pharmaceuticals, Jeddah, Saudi Arabia \\ ${ }^{4}$ College of Pharmacy, University of Petra, Amman, Jordan \\ Email: o.najib@iprc.com.jo
}

\begin{abstract}
A randomized, two-way, crossover study was conducted in 34 fasting, healthy, male volunteers to compare the bioavailability of two brands of etoricoxib 120mg tablets; Etoricoxib-SAJA (Saudi Arabian Japanese Pharmaceutical Company, Saudi Arabia) as test and ARCOXIA® (Merck Sharp \& Dohme Ltd, UK) as reference product. The study was performed at the International Pharmaceutical Research Centre (IPRC), Amman, Jordan. The drug was administered with $240 \mathrm{ml}$ of water after a 10-h overnight fasting on two treatment days separated by 2 -weeks washout period.

After oral dosing, serial blood samples were collected for a period of $72 \mathrm{~h}$. Plasma harvested from blood was analyzed for etoricoxib by validated HPLC method with UV-visible detector capable to detect etoricoxib in the range of $30-3000 \mathrm{ng} / \mathrm{ml}$ with limit of quantitation of $30.0 \mathrm{ng} / \mathrm{ml}$. The pharmacokinetic parameters including truncated $\mathrm{AUC}_{0 \rightarrow 72}$, Cmax and Tmax were determined from plasma concentrations of both formulations and found to be in good agreement with reported values. Truncated $\mathrm{AUC}_{0 \rightarrow 72}$ and $\mathrm{Cmax}$ were tested for bioequivalence after log-transformation of data.

No significant difference was found based on ANOVA; 90\% confidence interval $95.72-102.48 \%$ for $\mathrm{AUC}_{0 \rightarrow 72}, 89.76-106.81 \%$ for Cmax of test/reference ratio for these parameters were found within bioequivalence acceptance range of $80-125 \%$. Based on these statistical inferences, it was concluded that Etoricoxib-SAJA is bioequivalent to ARCOXIA®.
\end{abstract}

Keywords: Etoricoxib, bioequivalence, pharmacokinetics.

\section{Introduction}

Bioequivalence of two formulations of the same drug comprises equivalence with respect to the rate and extent of their absorption. The area under concentration time curve (AUC) generally serves as the characteristic of the extent of absorption while the peak concentration $\left(\mathrm{C}_{\max }\right)$ and the time of its occurrence $\left(T_{\max }\right)$, reflect the rate of absorption, especially in fast releasing drug formulations [1,2]. The present study was conducted to evaluate the bioequivalence of two brands of Etoricoxib $120 \mathrm{mg}$ tablets in fasting, healthy human volunteers.

Etoricoxib is an NSAID with a molecular weight of $358.8419 \mathrm{~g} / \mathrm{mol}$, a molecular formula of $\mathrm{C}_{18} \mathrm{H}_{15} \mathrm{ClN}_{2} \mathrm{O}_{2} \mathrm{~S}$ and partition coefficient (XLogP) of 3.9, and it exists in various crystalline polymorphs. It is highly selective cyclooxygenase 2 (COX-2) inhibitor with anti-inflammatory and analgesic properties. The selectivity of etoricoxib for COX-2 over COX-1 can be seen through the selectivity ratio (COX-1/COX-2) where etoricoxib has a value of 106 in human plasma. Etoricoxib is an oral, selective cyclo-oxygenase-2 (COX-2) inhibitor within the clinical dose range. Across clinical pharmacology studies, etoricoxib produced dose-dependent inhibition of COX-2 without inhibition of COX-1 at doses up to 150 mg daily. Etoricoxib did not inhibit gastric prostaglandin synthesis and had no effect on platelet function. Cyclooxygenase is responsible for generation of prostaglandins. Two isoforms, COX-1 and COX-2, have been identified. COX-2 is the isoform of the enzyme that has been shown to be induced by pro-inflammatory stimuli and has been postulated to be primarily responsible for the synthesis of prostanoid mediators of pain, inflammation, and fever. COX-2 is also involved in ovulation, 
implantation and closure of the ductus arteriosus, regulation of renal function, and central nervous system functions (fever induction, pain perception and cognitive function). It may also play a role in ulcer healing. COX-2 has been identified in tissue around gastric ulcers in man but its relevance to ulcer healing has not been established.

Orally administered etoricoxib is well absorbed. The absolute bioavailability is approximately $100 \%$. Following $120 \mathrm{mg}$ once-daily dosing to steady state, the peak plasma concentration (geometric mean $\left.\mathrm{C}_{\max }=3.6 \mathrm{\mu g} / \mathrm{ml}\right)$ was observed at approximately 1 hour $\left(\mathrm{T}_{\max }\right)$ after administration to fasted adults. The geometric mean area under the curve $\left(\mathrm{AUC}_{0-24 \mathrm{hr}}\right)$ was $37.8 \mu \mathrm{g} \bullet \mathrm{hr} / \mathrm{ml}$. The pharmacokinetics of etoricoxib is linear across the clinical dose range. Dosing with food (a high-fat meal) had no effect on the extent of absorption of etoricoxib after administration of a 120-mg dose. The rate of absorption was affected, resulting in a $36 \%$ decrease in $\mathrm{C}_{\max }$ and an increase in $\mathrm{T}_{\max }$ by 2 hours. These data are not considered clinically significant. In clinical trials, etoricoxib was administered without regard to food intake.

Etoricoxib is approximately $92 \%$ bound to human plasma protein over the range of concentrations of 0.05 to $5 \mathrm{\mu g} / \mathrm{ml}$. The volume of distribution at steady state $\left(\mathrm{V}_{\mathrm{dss}}\right)$ was approximately $120 \mathrm{~L}$ in humans. Etoricoxib is extensively metabolised with $<1 \%$ of a dose recovered in urine as the parent drug. The major route of metabolism to form the 6'-hydroxymethyl derivative is catalyzed by CYP enzymes. CYP3A4 appears to contribute to the metabolism of etoricoxib in vivo. In vitro studies indicate that CYP2D6, CYP2C9, CYP1A2 and CYP2C19 also can catalyse the main metabolic pathway, but their quantitative roles in vivo have not been studied. Five metabolites have been identified in man. The principal metabolite is the $6^{\prime}$-carboxylic acid derivative of etoricoxib formed by further oxidation of the 6'-hydroxymethyl derivative. These principal metabolites either demonstrate no measurable activity or are only weakly active as COX-2 inhibitors. None of these metabolites inhibit COX-1.

Following administration of a single 25-mg radiolabeled intravenous dose of etoricoxib to healthy subjects, $70 \%$ of radioactivity was recovered in urine and $20 \%$ in feces, mostly as metabolites. Less than $2 \%$ was recovered as unchanged drug. Elimination of etoricoxib occurs almost exclusively through metabolism followed by renal excretion. Steady state concentrations of etoricoxib are reached within seven days of once daily administration of $120 \mathrm{mg}$, with an accumulation ratio of approximately 2 , corresponding to a half-life of approximately 22 hours. The plasma clearance after a 25-mg intravenous dose is estimated to be approximately $50 \mathrm{ml} / \mathrm{min}$. Etoricoxib is indicated in adults and adolescents 16 years of age and older for the symptomatic relief of osteoarthritis (OA), rheumatoid arthritis (RA), ankylosing spondylitis, and the pain and signs of inflammation associated with acute gouty arthritis. Etoricoxib is indicated in adults and adolescents 16 years of age and older for the short-term treatment of moderate pain associated with dental surgery.

\subsection{Objectives of the Study}

The purpose of this study was to determine the bioequivalence of a new tablet formulation of etoricoxib (Etoricoxib-SAJA) produced locally in Saudi Arabia by Saudi Arabian Japanese Pharmaceutical Company, in comparison with ARCOXIA® from Merck Sharp \& Dohme Ltd, UK.

\section{Material and Methods}

\subsection{Study Products}

Test Product Etoricoxib-SAJA $120 \mathrm{mg}$ tablets

Batch No. 15D023ET, Expiry 04/2017

Manufacturer Saudi Arabian Japanese Pharmaceutical Company, Saudi Arabia

Reference Product ARCOXIA® 120 mg tablets

Batch No. J004987, Expiry 12/2015

Manufacturer Merck Sharp \& Dohme Ltd, UK

To ensure that the reproduction of your illustrations is of a reasonable quality, we advise against the use of shading. The contrast should be as pronounced as possible. 


\subsection{Study Subjects}

Thirty-four (34) healthy adult male volunteers participated in this study at the International Pharmaceutical Research Centre (IPRC), Amman, Jordan. The mean age was $30 \pm 5.64$ years with a range of 19 - 40 years, mean body weight was $75 \pm 10.74 \mathrm{~kg}$ with a range of $54-96 \mathrm{~kg}$ and mean height was $175 \pm 5.92 \mathrm{~cm}$ with a range of $162-190 \mathrm{~cm}$. On the basis of medical history, clinical examination and laboratory investigation (hematology, blood biochemistry, and urine analysis), no subject had a history or evidence of hepatic, renal, gastrointestinal or hematologic deviations or any acute or chronic diseases or allergy to the drug under investigation or any of its ingredients. Consumption of alcohol or beverages or food containing methylxanthines was not permitted for the volunteers $48 \mathrm{~h}$ prior to the study and after drug administration until the last blood sample was collected in the respective study phase. The subjects were instructed to abstain from taking any medication for at least 1 week prior to and during the study period. Informed consent was obtained from the subjects after explaining the nature and purpose of the study. The study protocols were approved by the Institutional Review Board (IRB) of International Pharmaceutical Research Centre (IPRC), Amman-Jordan.

\subsection{Study Design}

This study was based on a single dose, randomized, two treatment, two period crossover design. On the morning of phase I, after an overnight fasting $(10 \mathrm{~h})$ volunteers were given single dose of either formulation (reference or test) of etoricoxib with $240 \mathrm{ml}$ of water. Lunch and dinner were served at 4 and $12 \mathrm{~h}$, respectively, after drug administration. Volunteers were ambulatory during the study but prohibited from strenuous activity. Approximately, $8 \mathrm{ml}$ of blood samples for etoricoxib assay were drawn through indwelling cannula before $(0 \mathrm{~h})$ and at $0.25,0.50,0.75,1.00,1.25,1.50,1.75,2.00,2.50$, $3.00,4.00,6.00,8.00,12.00,16.00,24.00,36.00,48.00,60.00$ and $72.00 \mathrm{~h}$ after dosing. The blood samples were collected in glass tubes containing heparin, and centrifuged at $3500 \mathrm{rpm}$ for $10 \mathrm{~min}$; plasma was separated and kept frozen at $-20^{\circ} \mathrm{C}$ in properly labeled tubes. After a period of 14 days the study was repeated in the same manner to complete the crossover design.

\subsection{Drug Assay}

All plasma samples were analysed for Etorocoxib concentrations according to a sensitive, selective and accurate high performance liquid chromatography (HPLC) method with UV detection.

The chromatographic separation and quantitative determination were performed using Waters Alliance 2695 HPLC system coupled with Waters 2489 UV detector and controlled by Waters Empower software. Plasma samples were extracted with tert-butyl methyl ether after the internal standard and sodium carbonate had been added. After vortex-mixing followed by centrifugation, the organic phase was transferred into a new tube and evaporated to dryness in water bath at a temperature of $45^{\circ} \mathrm{C}$ under a gentle stream of nitrogen gas. Chromatographic separation was achieved using Symmetry C8 HPLC column from Waters. The mobile phase consisted of de-ionized water, acetonitrile, and methanol. The mobile phase was eluted at a flow rate of $1.5 \mathrm{ml} / \mathrm{min}$ at $35^{\circ} \mathrm{C}$. UV detection of Etorecoxib and the internal standard was performed at $280 \mathrm{~nm}$. The calibration curves were linear over a range of 30-3000 $\mathrm{ng} / \mathrm{ml}$ using $500 \mathrm{pl}$ plasma samples.

\subsection{Pharmacokinetic and Statistical Analysis}

Pharmacokinetic analysis was performed by means of model independent method using Kinetica5.1 SP1 computer program [3], Area under the curve to the last measurable concentration $\left(\mathrm{AUC}_{0-72}\right)$ was calculated by the linear trapezoidal rule.

For the purpose of bioequivalence analysis $\mathrm{AUC}_{0-72}$ and $\mathrm{Cmax}$ were considered as primary variables. Two-way analysis of variance (ANOVA GLM procedure; Kinetica5.1 SP1 Computer program) for crossover design was used to assess the effect of formulations, periods, sequences and subjects on these parameters. Difference between two related parameters was considered statistically significant for pvalue equal to or less than 0.05. Parametric 90\% confidence intervals [4], based on the ANOVA of the mean test/reference $(\mathrm{T} / \mathrm{R})$ ratios of $\mathrm{AUCs}$ and $\mathrm{C}_{\max }$ were computed (3-7). 


\section{$3 \quad$ Results and Discussion}

Etoricoxib was well tolerated by the volunteers; unexpected incidents that could have influenced the outcome of the study did not occur. There was no drop-out and all volunteers who started the study continued to the end and were discharged in good health.

The described analytical method was proven to be sensitive and accurate for determination of etoricoxib plasma concentration. Retention times were 6.5 and $8.6 \mathrm{~min}$ for Rofecoxib (internal standard) and etoricoxib, respectively.

Under the described conditions, the lower limit of quantitation was $30.0 \mathrm{ng} / \mathrm{ml}$. The relationship between concentration and peak area ratio was found to be linear within the range of 30.0-3000.0 ng/ml. Stability study showed that etoricoxib was stable in plasma for 6 weeks when stored at $-20^{\circ} \mathrm{C}$.

Both formulations were readily absorbed from the gastrointestinal tract and etoricoxib was measurable at the first sampling time $(0.33 \mathrm{~h})$ in all 24 volunteers. The mean concentration-time profile of the two formulations is shown in the Figure 1 indicating that the mean plasma drug concentration profiles of the two brands were closely similar and superimposable. Peak concentration was attained at 1.34 and $1.26 \mathrm{~h}$ after drug administration and was still detectable up till $72 \mathrm{~h}$ for most volunteers. All calculated pharmacokinetic parameter were in good agreement with reported values. Table 1 shows the pharmacokinetic parameters for the two brands of etoricoxib $120 \mathrm{mg}$ tablets.

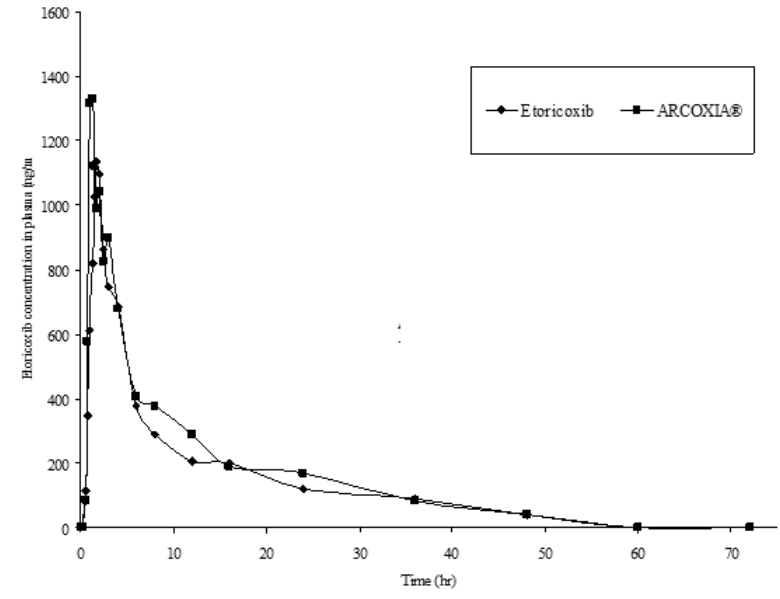

Figure 1 Mean plasma concentration of etoricoxib 120mg tablets after oral administration of single dose of two brands to 34 healthy human volunteers

The extent of absorption is a key characteristic of drug formulation, and therefore AUC is an important parameter for comparative bioavailability (bioequivalence) study. However, the other two parameters, $\mathrm{C}_{\max }$ and $\mathrm{T}_{\max }$, are also important features of the plasma level profile and could affect the therapeutic use of a drug and hence were also considered in the study. The relative bioavailability of Etoricoxib-SAJA was $99.04 \%$ for $\mathrm{AUC}_{0-72}$, and $97.91 \%$ for $\mathrm{C}_{\max }$.

The most important objective of bioequivalence testing is to assure the safety and efficacy of generic formulations. When two formulations of the same drug are equivalent in the rate and extent to which the active drug becomes available to the site of drug action, they are bioequivalent and thus consider therapeutically equivalent. To demonstrate bioequivalence certain limits should be set depending on the nature of drug, patient population, and clinical end points. It is generally accepted that for basic pharmacokinetic characteristics, such as $\mathrm{AUC}$ and $\mathrm{C}_{\max }$, the standard equivalence range is $0.8-1.25$. The results of pharmacokinetic and statistical analysis are shown in Tables 1, 2 and 3. 
Table 1. Pharmacokinetic parameters of etoricoxib tablets (Mean $\pm S D ; n=34$ )

\begin{tabular}{c|c|c}
\hline \multirow{2}{*}{ Pharmacokinetic Parameter } & \multicolumn{2}{|c}{ Treatment (Mean \pm SD) } \\
\cline { 2 - 3 } & TEST Product & REFERENCE Product \\
\hline $\mathbf{C}_{\max }(\mathbf{n g} / \mathbf{m l})$ & $1923.900 \pm 466.83$ & $1986.143 \pm 614.41$ \\
truncated AUC $\mathbf{0}_{\mathbf{0} 7 \mathbf{7}}(\mathbf{n g} . \mathbf{h} / \mathbf{m l})$ & $23067.3 \pm 8978.36$ & $23478.2 \pm 9719.32$ \\
$\mathbf{T}_{\max }(\mathbf{h})$ & $1.34 \pm 0.65$ & $1.26 \pm 0.77$ \\
\hline
\end{tabular}

Table 2. Bioequivalence mertics of etoricoxib tablets

\begin{tabular}{c|c|c|c}
\hline \multirow{2}{*}{ Pharmacokinetic Parameter } & \multicolumn{3}{|c}{ 90\% Confidence intervals of parametric means } \\
\cline { 2 - 4 } & Point estimate \% & Lower Limit \% & Upper Limit \% \\
\hline $\mathbf{C}_{\max }$ & 97.91 & 89.76 & 106.81 \\
truncated $\mathbf{A U C}_{0 \rightarrow 72}$ & 99.04 & 95.72 & 102.48 \\
\hline
\end{tabular}

Table 3. ANOVA of etoricoxib tablets

\begin{tabular}{c|c|c|c|c}
\hline Pharmacokinetic Parameter & Period & Subject (sequence) & Drug & Sequence \\
\hline \multicolumn{5}{c}{ Transformed to natural logarithm (neperian) } \\
\hline $\mathbf{A U C}_{0-72}(\mathrm{~N}=\mathbf{3 4})$ & $9.22 \times 10^{-1}$ & $6.45 \times 10^{-19}$ & $6.37 \times 10^{-1}$ & $2.32 \times 10^{-10}$ \\
\hline $\mathbf{C}_{\max }(\mathrm{N}=\mathbf{3 4})$ & $7.72 \times 10^{-1}$ & $9.91 \times 10^{-3}$ & $6.85 \times 10^{-1}$ & $6.41 \times 10^{-1}$ \\
\hline \multicolumn{5}{c}{ Untransformed (Original) } \\
\hline $\mathrm{AUC}_{\mathbf{0}-72}(\mathrm{~N}=\mathbf{3 4})$ & $7.80 \times 10^{-1}$ & $5.30 \times 10^{-17}$ & $4.46 \times 10^{-1}$ & $2.15 \times 10^{-11}$ \\
\hline $\mathbf{C}_{\max }(\mathrm{N}=\mathbf{3 4})$ & $8.24 \times 10^{-1}$ & $2.00 \times 10^{-2}$ & $5.68 \times 10^{-1}$ & $6.94 \times 10^{-1}$ \\
\hline $\mathbf{t}_{\max }(\mathrm{N}=\mathbf{3 4})$ & $3.89 \times 10^{-1}$ & $3.29 \times 10^{-2}$ & $5.76 \times 10^{-1}$ & $3.89 \times 10^{-1}$ \\
\hline
\end{tabular}

The mean and standard deviation of $\mathrm{AUC}_{0-72}$ and $\mathrm{C}_{\max }$ of the two products did not differ significantly, suggesting that the blood profiles generated by Etoricoxib-SAJA are comparable to those produced by ARCOXIA®. Analysis of variance (ANOVA) for these parameters, after log-transformation of the data, showed no statistically significant difference between the two formulations either in periods, formulations or sequence, having $\mathrm{p}$ value greater than 0.05. Ninety percent confidence intervals also demonstrated that the ratios of $\mathrm{AUC}_{0-72}$ or $\mathrm{C}_{\max }$, of the two formulations lie within the FDA acceptable range of $80-125 \%$. For $\mathrm{T}_{\max }$ the parametric point estimate of difference (test-reference) was $\pm 0.08 \mathrm{~h}$, and found to be within the acceptance limits $( \pm 20 \%$ of reference mean). Plasma levels may be used as surrogate parameters for clinical activity; therefore results of this study suggest equal clinical efficacy of the two brands of etoricoxib.

\section{Conclusion}

Statistical comparison of the $\mathrm{AUC}_{0-72}$ and $\mathrm{Cmax}$ clearly indicated no significant difference between Etoricoxib-SAJA and ARCOXIA® tablets in any of the calculated pharmacokinetic parameters. The confidence intervals for the ratios of mean $\mathrm{AUC}_{0-72}$ and $\mathrm{Cmax}$ indicated that these values were entirely within the bioequivalence acceptance range of $80-125 \%$ (using log-transformed data). Based on the above we can conclude that Etoricoxib-SAJA, manufactured by Saudi Arabian Japanese Pharmaceutical Company, Saudi Arabia is bioequivalent to ARCOXIA®, manufactured by Merck Sharp \& Dohme Ltd, $\mathrm{UK}$, and that both products can be considered equally effective in medical practice.

Acknowledgments. We thank SAJA Pharmaceutical for funding this study. We are grateful to the staff at IPRC for the administration of the study protocol and data collection. We are grateful to the volunteers who participated in this study. 


\section{References}

1. Hauschke D, Steinijans VW, Diletti E. A distribution-free procedure for the statistical analysis of bioequivalence studies. Int J Clin Pharmacol Ther Toxicol 1990; 28: 72-78.

2. Schulz HU, Steinijans VW. Striving for standards in bioequivalence assessment: a review. Int J Clin Pharmacol Ther Toxicol 1992; 30(Suppl.1): S1-S6.

3. Kinetica, Version 5.1SP1, User Manual.

4. Guidance for Industry: Bioavailability and Bioequivalence Studies for Orally Administered Drug Products General Considerations. March 2003. Division of Drug Information, Center for Drug Evaluation and Research, Food and Drug Administration 5600 Fishers Lane Rockville, MD 20857, USA. 